\title{
Parámetros productivos de cuyes G en la costa central del Perú
}

\author{
Productive Parameters of G guinea Pigs in the central coast of Peru
}

\author{
Graciela Yamada A. ${ }^{1}$, Víctor Bazán R., ${ }^{1,2}$ Nadia Fuentes N. ${ }^{1}$
}

\section{Resumen}

\begin{abstract}
El objetivo de la investigación fue determinar los parámetros productivos de cuyes $\mathrm{G}$ a nivel de la costa central del Perú. Se recolectó información de datos productivos de 180 partos de 39 cuyes $\mathrm{G}$ reproductoras con 452 crías destetadas a los 16.3 días en promedio entre abril de 2016 y mayo de 2017 en la estación experimental de Huaral del C.I. IVITA de la Universidad Nacional Mayor de San Marcos. Se evaluó el número de nacidos $(\mathrm{NN})$, números de nacidos vivos (NNV), peso de las crías al nacimiento $(\mathrm{PCrN})$, peso de la camada al nacimiento (PCN), número de destetados (ND), peso de las crías al destete (PcrD), peso de la camada al destete (PCD) y tasa de mortalidad (M). Los resultados fueron: $\mathrm{NN}$ de $2.82 \pm 1.14$ crías por parto ( $1-5$ crías), $\mathrm{NNV}$ de $2.80 \pm 1.13$ crías, $\mathrm{PCrN}$ de $139.3 \pm 30.9$ g, PCN de $389.6 \pm 121.3$ g, ND de $2.58 \pm 1.02$ crías, PCrD de $248.0 \pm 62.3$ g, PCD de $640.6 \pm 211.9$ g y M de $8.89 \%$. Solo se encontró diferencia significativa en $\mathrm{PCrD}$ por efecto del número de partos $(\mathrm{p}<0.05)$.
\end{abstract}

Palabras clave: cuy G; parámetros productivos; peso de crías; nacimientos

\section{AbSTRACT}

The objective of this study was to determine the productive parameters of guinea pigs of the $\mathrm{G}$ lineage at the level of the central coast of Peru. Information on productive data was collected from 180 parturitions of 39 breeding guinea pigs and 452 weaned individuals at 16.3 days between April 2016 and May 2017 from the Huaral experimental station of the IVITA Research Centre of the National University of San Marcos. The number of births (NN), number of live births (NNV), body weight at birth (PCrN), litter weight at birth (PCN), number of weaned animals (ND), body weight at weaning (PcrD),

\footnotetext{
${ }^{1}$ Estación Experimental Huaral, Centro de Investigación IVITA, Facultad de Medicina Veterinaria, Universidad Nacional Mayor de San Marcos, Lima, Perú

${ }^{2}$ E-mail:vbazanr@unmsm.edu.pe
}

Recibido: 13 de septiembre de 2017

Aceptado para publicación: 24 de marzo de 2018 
litter weight at weaning (PCD) and mortality rate (M). The results were: NN $2.82 \pm 1.14$ pups per parturition (1-5 pups), NNV $2.80 \pm 1.13$ pups, $\mathrm{PCrN} 139.3 \pm 30.9 \mathrm{~g}, \mathrm{PCN} 389.6 \pm$ $121.3 \mathrm{~g}, \mathrm{ND} 2.58 \pm 1.02$ pups, PCrD $248.0 \pm 62.3 \mathrm{~g}, \mathrm{PCD} 640.6 \pm 211.9 \mathrm{~g}$ and M $8.89 \%$. Only $\mathrm{PCrD}$ showed significant difference due to the number of parturitions $(\mathrm{p}<0.05)$.

Key words: G guinea pigs; productive parameters; birth weight; births

\section{INTRODUCCIÓN}

El cuy es un roedor muy dócil y de fácil manejo. Las ventajas de la crianza de cuyes incluyen su calidad de especie herbívora, su ciclo reproductivo corto, la facilidad de adaptación a diferentes ecosistemas y una alimentación versátil, que utiliza insumos no competitivos con la alimentación de otros monogástricos (Chauca, 1997). El cuy presenta un gran potencial de producción gracias a sus características de gran capacidad para el consumo de forraje, alta precocidad y aptitud para la producción de carne (Burgos et al., 2010).

El Perú es el primer país productor de cuyes en la región, donde su explotación se ha ido incrementado en forma notable, incluyendo el mejoramiento genético para lograr animales con mayor conformación cárnica y mayor número de crías por parto. En la Estación Experimental de El Mantaro, Junín, del Centro de Investigación IVITA, Universidad Nacional Mayor de San Marcos se ha logrado obtener cuyes $\mathrm{G}$ mejorados genéticamente a nivel de la sierra (3300 msnm), una línea cárnica mejorada obtenida de cuatro líneas, dos paternas (precoz y cárnica) y dos maternas (prolífica y lechera), los cuales están especializados en producción de carne con altos índices de productividad (Romero, 2014).

Según Rodríguez et al. (2015), una de las características productivas de importancia económica se relaciona con la madre, pues considera el total de crías nacidas, el número de parto, el efecto genético y el sistema de empadre. Así mismo, señalan que el número de destetados es una variable influenciada por la habilidad materna y el número de partos de la madre, estación del año al parto, edad al destete, tamaño de la camada al nacimiento y mortalidad de las crías, en tanto que para la variable peso de la camada al nacimiento y al destete se presenta el efecto del tamaño de la camada, número de partos y la habilidad materna. El objetivo del presente estudio fue determinar los parámetros productivos de los cuyes $\mathrm{G}$ a nivel de la costa central del Perú.

\section{Materiales y Métodos}

El trabajo se desarrolló en el Módulo Demostrativo de Cuyes de la Estación Huaral del Centro de Investigación (CI) IVITA de la Universidad Nacional Mayor de San Marcos (UNMSM), ubicado en el km 6.5 de la carretera Chancay-Huaral, distrito de Huaral, Lima, Perú, a una altura de $200 \mathrm{msnm}$. La temperatura promedio anual es de $19.4{ }^{\circ} \mathrm{C}$ con $16 \mathrm{~mm}$ de precipitación pluvial media anual y humedad relativa de $82.3 \%$.

Se emplearon cuyes $\mathrm{G}$ provenientes de la Estación Experimental El Mantaro del CI IVITA, UNMSM. Las instalaciones cuentan con un galpón de material noble con ventanas amplias y techo a dos aguas con láminas de Aluzinc. Las jaulas de reproducción son de alambre electrosoldado de 1.6x1.0x0.4 m. La altura de piso a jaula es de $0.50 \mathrm{~m}$. 
El núcleo reproductor consta de un macho con seis hembras. El empadre se inicia a partir de los tres meses con pesos vivos superiores de $750 \mathrm{~g}$ en hembras y a partir de los 4 meses con pesos superiores a $1 \mathrm{~kg}$ en machos. Se utiliza el empadre continuo para aprovechar el celo posparto. La alimentación es a base de maíz chala forrajero y afrecho de trigo, además de agua ad libitum.

Los datos se recolectaron entre abril de 2016 y mayo de 2017 de un total de 180 partos de 39 cuyes $G$ reproductoras y 452 crías $\mathrm{G}$ destetadas a los 16.3 días en promedio. Los pesos semanales se tomaron en una balanza digital con una precisión de $10 \mathrm{~g}$. Los datos se analizaron mediante un análisis de varianza simple, empleando el paquete estadístico IBM SPSS Statistics v. 22, utilizando un nivel de significancia de $\mathrm{p}<0.05$.

\section{Resultados y Discusión}

El número de nacidos $(\mathrm{NN})$ fue de 2.82 \pm 1.14 crías (Cuadro 1), siendo valores similares a los reportados para cuyes Perú con 2.87 crías (Chauca, 1997). Valores menores (2.60 crías) fueron reportados por Muscari et al. (1994), mientras que Rodríguez et al. (2015) obtuvieron 3.21 crías en cuyes Andina, Chauca (1997) obtuvo 3.48 crías en cuyes mejorados y Muscari et al. (2004) reportaron 3.35 crías en cuyes Perú.

El número de nacidos vivos (NNV) fue de $2.80 \pm 1.13$ crías (Cuadro 1), valores superiores al de 2.30 crías reportados por Muscari et al. (1994) y Guevara et al. (2015) en cuyes Perú y Cieneguilla, respectivamente. Por otro lado, Rodríguez et al. (2015) reportaron 2.92 crías en cuyes Andina y Chauca (1997) obtuvieron 2.95 crías en cuyes mejorados. El número de destetados fue de $2.58 \pm 1.02$ crías (Cuadro 1), superior al 2.34 \pm 1.08 reportado por Rodríguez et al. (2015), pero inferior al 2.8 crías reportado por Chauca et al. (1997).
El peso de las crías al nacimiento fue de $139.3 \pm 30.9 \mathrm{~g}$ (Cuadro 1). Otros valores reportados en la literatura son de $115 \mathrm{~g}$ en crías Andina (Muscari et al., 2004), $121 \mathrm{~g}$ en crías Perú (Chauca, 1997), 128.0 $\pm 29.1 \mathrm{~g}$ en cuyes Inti (Chauca et al., 2013), $176 \mathrm{~g}$ en crías Perú (Atacusi, 2015) y 179 g en crías Cieneguilla (Guevara et al., 2015). Así mismo, el peso de las crías al nacimiento fue ligeramente superior en cuyes de segundo y tercer parto, pero sin que se hayan encontrado diferencias significativas por efecto del número de parto.

El peso de los machos al nacimiento fue de $140.1 \pm 32.5 \mathrm{~g}$ y de las hembras fue de $138.3 \pm 28.8 \mathrm{~g}$, sin diferencias significativas entre sexos (Cuadro 1). Higaonna et al. (2013) obtuvo pesos al nacimiento en cuyes Merino de $142.3 \pm 34.3 \mathrm{~g}$ en machos y de $141.0 \pm 17.2 \mathrm{~g}$ en hembras, sin diferencia significativa entre sexos, a diferencia de Rodríguez et al. (2013), quienes reportaron pesos mayores para los machos, en tanto que Muscari et al. (2013) reportaron pesos mayores para las hembras. Por otro lado, el peso de la camada al nacimiento fue de $389.6 \pm$ $121.3 \mathrm{~g}$, en comparación con los $270.5 \mathrm{~g}$ reportados por Chauca (1997) y $431.8 \pm 154.5 \mathrm{~g}$ reportado por Rodríguez et al. (2015).

El peso de las crías al destete fue de $248.0 \pm 62.3 \mathrm{~g}$ (Cuadro 1), mientras que Muscari et al. (2004) reportan $202 \mathrm{~g}$ en cuyes Andina, Chauca et al. (2013) reportan 245.6 $\pm 57.4 \mathrm{~g}$ en cuyes Inti, y Chauca (1997) y Rodríguez et al. (2013) reportan $310 \mathrm{~g}$, en ambos casos, en cuyes mejorados.

El peso de las crías macho al destete fue de $248.9 \pm 64.9$ y en las hembras de 246.9 \pm 59.0 , sin diferencia significativa entre sexos. No obstante, Higaonna et al. (2013) trabajando con la raza Merino obtienen $256.7 \pm$ 44.8 en hembras y $270.4 \pm 62.7$ en machos; es decir, los machos fueron $5.1 \%$ más pesados que las hembras. Asimismo, Muscari et al. (2013) en cuyes Inti obtuvieron $282 \pm 38$ 
Cuadro 1. Parámetros productivos de cuyes G en la costa central del Perú

\begin{tabular}{lrrrr}
\hline \multicolumn{1}{c}{ Variables } & Promedio & d.e. & Machos $^{1}$ & Hembras \\
\hline Número de nacidos/camada & 2.82 & 1.14 & & \\
Número de nacidos vivos/camada & 2.80 & 1.13 & & \\
Número de destetados/camada & 2.58 & 1.02 & & \\
Peso de la camada al nacimiento $(\mathrm{g})$ & 389.6 & 121.3 & & \\
Peso de la camada al destete $(\mathrm{g})$ & 640.6 & 211.9 & & \\
Peso de las crías al nacimiento $(\mathrm{g})$ & 139.3 & 30.9 & $140.1 \pm 32.5$ & $138.3 \pm 28.8$ \\
Peso de las crías al destete (g) & 248.0 & 62.3 & $248.9 \pm 64.9$ & $246.9 \pm 59.0$ \\
Mortalidad (\%) & 8.89 & & & \\
\hline
\end{tabular}

${ }^{1}$ Sin diferencia significativa entre sexos

Cuadro 2. Peso vivo de las crías al destete en cuyes $\mathrm{G}$ en la costa central del Perú

\begin{tabular}{ccc}
\hline $\begin{array}{c}\text { Número de } \\
\text { parto }\end{array}$ & $\begin{array}{c}\text { Crías } \\
(\mathrm{n})\end{array}$ & Media \pm d.e. \\
\hline 1 & 100 & $216.8 \pm 49.7^{\mathrm{a}}$ \\
2 & 95 & $253.2 \pm 58.1^{\mathrm{b}}$ \\
3 & 105 & $249.5 \pm 47.0^{\mathrm{b}}$ \\
4 & 90 & $242.3 \pm 63.6^{\mathrm{b}}$ \\
5 & 62 & $296.3 \pm 76.8^{\mathrm{c}}$ \\
\hline a,b,c Superíndices diferentes dentro de columnas \\
indican diferencia estadística (p<0.05)
\end{tabular}

y $280 \pm 47 \mathrm{~g}$ en machos y hembras, respectivamente, sin deferencias significativas por efecto del sexo.

El peso de la camada al destete fue de $640.6 \pm 211.9 \mathrm{~g}$ (Cuadro 1). Otros valores reportados en la literatura son de $540 \mathrm{~g}$ en cuyes Perú (Muscari et al.,1994), $512.2 \mathrm{~g}$ (Chauca, 1997) y $691.7 \pm 329.4 \mathrm{~g}$ (Rodríguez et al., 2013).

El porcentaje de mortalidad fue de $8.9 \%$, valor inferior al $10 \%$ de muertes de crías lactantes reportados en CARE (2010). Chauca (1997) señala que durante la lactancia se presentan los más altos porcentajes de mortalidad, pudiendo llegar en crianzas familiares hasta $38-56 \%$ y en crianzas tecnificadas hasta $23 \%$.

El tamaño de la camada al nacimiento y número de nacidos vivos presentó un rango de 1 a 5 crías por parto, siendo mayores en el tercer parto, aunque sin diferencias significativas por número de parto. Rodríguez et al. (2015), en forma similar, no encontraron diferencias significativas en el número de crías nacidas según el número de parto, mientras que Muscari et al. (2004) encontraron diferencias entre el primer y el segundo parto en cuyes Andina $(p<0.05)$.

El peso de la camada al nacimiento se incrementó hasta el tercer parto para luego disminuir al cuarto y quinto parto; sin embargo, sin diferencias significativas por número de parto. Estos resultados difieren del trabajo de Rodríguez et al. (2015) en cuyes mejorados de la granja Cieneguilla de la Universidad Nacional Agraria La Molina, donde presentaron mayor peso las camadas del quinto parto $(\mathrm{p}<0.01)$.

El número de destetados por camada fue relativamente mayor en el tercer parto y menor en el quinto parto, pero sin que hubiera diferencias significativas por número de parto (Cuadro 2). El peso de las crías al des- 
tete fue mayor en madres de quinto parto $y$ menor en aquellas de primer parto $(\mathrm{p}<0.05)$. Por otro lado, el peso de la camada al destete siguió la misma tendencia que el número de crías destetadas, pero sin presentar diferencias significativas por efecto del número de parto, a diferencia del trabajo de Rodríguez et al. (2015) quienes encuentran diferencias en los pesos de camada al destete por efecto del parto $(\mathrm{p}<0.01)$.

\section{Conclusiones}

- Se encontró diferencia significativa en el peso de las crías al destete por efecto del número de parto.

- No hubo diferencias estadísticas significativas por efecto del número de parto en relación al número de nacidos, números de nacidos vivos, peso de las crías al nacimiento, peso de la camada al nacimiento, número de destetados y peso de la camada al destete.

- La mortalidad en crías lactantes fue de $8.89 \%$.

\section{Literatura Citada}

1. Ataucusi S. 2015. Manejo técnico de la crianza de cuyes en la Sierra del Perú. Callao, Perú: Caritas del Perú. 42 p.

2. Burgos W, Solarte C, Cerón M. 2010. Efecto del tamaño de camada y número de parto en el crecimiento de cuyes $(\mathrm{Ca}$ via porcellus Rodentia: caviidae). Rev Lasallista Investig 7: 47-55.

3. CARE . 2010. Guía de producción de cuyes. 52 p. [Internet]. Disponible en: http://www.care.org.pe/wp-content/ uploads/2015/06/Guia-de-Produccionde-Cuyes1.pdf

4. Chauca L. 1997. Producción de cuyes (Cavia porcellus). Roma: Organización de la Naciones Unidas para la Agricultura y la Alimentación. [Internet]. Disponible en: http://www.fao.org/docrep/ W6562S/W6562S00.htm
5. Chauca L, Muscari J, Huamán M, Higaonna R. 2013. Comportamiento reproductivo de cuyes de la raza Inti. En: XXXVIII Reunión APPA. Lima: Asociación Peruana de Producción Animal.

6. Guevara J, Tapia N, Condorhuaman C, Díaz P, Carcelén F, Peña D. 2015. Efecto del probiótico nativo del cuy $(\mathrm{Ca}$ via porcellus) suplementado a las madres sobre el peso de las crías al nacimiento y al destete. Rev Per Quim Ing Quim 18: 73-77.

7. Higaonna $R$, Reynaga M, Muscari J, Chauca L. 2013. Crecimiento del cuy Merino en la costa central. En: XXXVIII Reunión APPA. Lima: Asociación Peruana de Producción Animal.

8. Muscari J, Chauca L, Higaonna R. 1994. Caracterización de una línea mejorada de cuyes: Perú. En: XVII Reunión APPA. Huancavelica: Asociación Peruana de Producción Animal.

9. Muscari J, Chauca L, Higaonna R. 2004. Características de la línea de cuyes Andina. En: XXVII Reunión APPA. Piura: Asociación Peruana de Producción Animal.

10. Muscari J, Chauca L, Higaonna R. 2013. Caracterización del cuy Inti durante la lactancia. En: XXXVIII Reunión APPA. Lima: Reunión Asociación Peruana de Producción Animal.

11. Rodríguez H, Palomino M, Hidalgo V, Gutierrez G. 2013. Efectos de factores fijos y al azar sobre el peso al nacimiento y al destete en cuyes de la Costa central del Perú. Rev Inv Vet Perú 24: 16-24. doi: 10.15381/rivep.v24i1.1647

12. Rodríguez H, Gutierrez G, Palomino M, Hidalgo V. 2015. Características maternales al nacimiento y destete en cuyes de la costa central del Perú. Rev Inv Vet Perú 26: 77-85. doi: 10.15381/ rivep.v26i1.10941

13. Romero L, Wilson, 2014. Efecto de zonas geográficas y de alimentación sobre los parámetros productivos de cobayos de las líneas mejoradas de costa y de sierra. Tesis de Magister. Lima: Univ. Nacional Mayor de San Marcos. 60 p. 